\title{
Detection and tracking of the hand in remote chess game system
}

\author{
Huibai Wang ${ }^{1, a}$, Ruiming Xing ${ }^{2, b^{*}}$ \\ ${ }^{12}$ North China University of Technology,Beijing,100144,China \\ awanghb@ncut.edu.cn, ${ }^{b} x y z 19901207 @ 163 . c o m$
}

Keywords: Camshift, Kalman filter, Hand-tracking, Hand-detection, chess game system

\begin{abstract}
Remote chess game system use the network transmission to let the rival chess image transfor to our own computer. At the same time, the virtual chess pieces that represent our own chess pieces are in the rival chess image.The image on monitor show the two sides of chess pieces real-timely.The detection and tracking of the hand has very important significance to detect the movement of chess pieces and the effect of the system.In this paper,an algorithm based on skin color detection and inter-frame difference is used to detect the position of hand and this position is the initial search window in Camshift.This algorithm can detect the position of hand automatically. Kalman filter is used to predict to solve the color interference question that may track the wrong position. The results show that this algorithm can detect and track the hand accurately and real-timely.
\end{abstract}

\section{Introduction}

Motion detection is an important research direction in computer vision, Widely used in monitoring system, vehicle tracking, medical, military, etc.Detection and tracking of hand has great significance in human-computer interaction, gesture recognition and other aspects.

In this paper, an algorithm based on skin color detection and Camshift is proposed, which can detect and track the position of hand automatically and quickly. Camshift algorithm is a moving object tracking algorithm that use color histogram to match, the algorithm has strong real-time and stability ${ }^{[7]}$, but this algorithm need manual initialize the search window at the start of the algorithm. Sometimes the moving object will track failure under color interference and some other situations. To deal with the problems in traditional Camshift algorithm, An algorithm based on inter-frame difference and skin color detection is proposed to initialize the search window automatically.and Kalman filter is used to predict to solve the color interference question ${ }^{[6]}$. The experiment results demonstrate that the algorithm can track and detect the hand accurately and has strong real-time.

\section{Detection of the hand}

As Camshift tracking algorithm need manual initialize the search window at the start of the algorithm. An algorithm based on inter-frame difference and skin color detection is used to detect hand and use its position to initialize the search window.

Skin color detection is a method that use the distribution features of skin color in color space to detect the skin color regions in image, The results of experiment show that skin color cluster together better in $\mathrm{YCbCr}$ color space and it is a non-linear function relation with the brightness ${ }^{[1]}$. In coordinate system of $\mathrm{CbCr}$, the skin color region approximate ellipse. Firstly, the color image is transformed into YCBCr color space. Then if we can attain the elliptical boundary equation of skin color region,we can know where is the skin color region in image. The alliptical is inclined.So we use KL transform to convert the coordinate system $^{[2]}$.In the coordinate system, The ellipse center is the origin of the coordinate system and the long and short axis of the ellipse is the coordinate axis, as shown in Fig $1^{[3]}$. We can only judge by if the pixel's value in image is in the elliptical boundary to detect the skin color region in image ${ }^{[2]}$. Skin color detection convert the initial picture into binary image.But in this binary 
image,Many color similar to the skin color is also identified as skin color. So we need to use inter-frame difference to eliminate the interference.

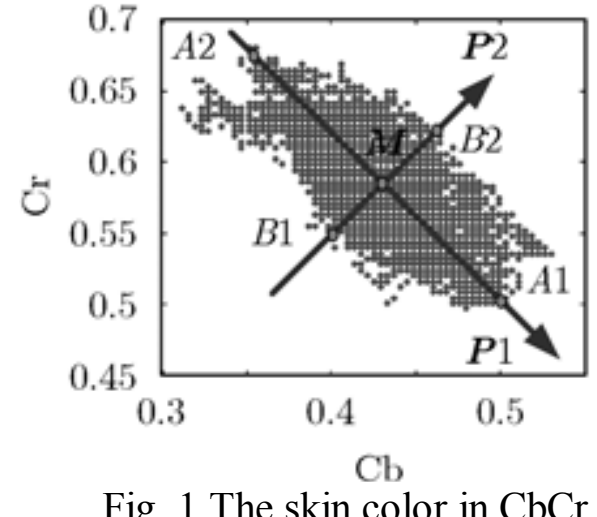

Every frame in the video is converted to binary image that is based on the skin color detection.First,save the first frame,then Then, each pixel of every frame behind it will be compared with each pixel of the first frame. When the pixel values are the same, this pixel will be recognized as the background image, when the pixel values are different, it will be recognized as the skin color, this area is recognized as the hand. This method will exclude other interference regions that the color is closer to the color of skin, but when the moving range of hand is small, there are many overlapping parts with the first frame in the hand movement area and small portion can be extracted about the hand .So, we set a threshold, if the area of the moving region is more than the threshold, this region is used to initialize the search window.

When the area of the moving area is larger than the threshold, the white areas in the binary image have the hand positon in the first frame and the current frame and error points due to the influence of light and other factors.Compared to the first frame can remove the skin region that is in the first frame.Then we can remove the error points by erode the binary image. The white area in the binary image is the position of hand in the frame, this area can be used as the initial search window of Camshift.

\section{Tracking of the Hand}

Camshift is an algorithm that is improved based on MeanShift. The initial searching window, of which the position is captured by hand detection, achieves the automatic tracking of hand.We can divide the process of hand tracking into the following steps:

(1).In order to reduce the effect of tracking result caused by the changes of light brightness. The image is converted to HSV color space according to a color histogram generated by the appearing frequency of $h$ vector in initial searching window. The more divided color distribution can obtain higher tracking accurate. The image can be converted to a color probability distribution by using the histogram.

(2).MeanShift is an optimal algorithm which is utilized to search local area using the gradient climb of probability density ${ }^{[4]}$. We can obtain the hand's position and size according to the repeatedly iterative calculation of MeanShift algorithm.

(3). The initial value of the next searching window frames is calculated by he obtained hand position and size of last frame using MeanShift algorithm. Then we can realize the continuous tracking of hand.

However, hand tracking is propably lost due to the color when the image have very similar color with hand, which will affect the accuracy of tracking. This paper introduces the Kalman filter to achieve the prediction of hand position and make a modification when hand tracking is lost, ensuring the tracking accuracy.

Kalman filter is a kind of time domain filter, which can calculate the forecast value of current state according to the forecast value of last moment and current observation value ${ }^{[5]}$. During the process of hand tracking, we utilize Camshift algorithm to calculate the position and size of hand. Meanwhile, we predict the hand position using Kalman filter and then update Kalman filter using the calculated position value by Camshift algorithm. According to the comparison of computed result between 
CamShift algorithm and Kalman filter, when the predicted position of Kalman filter is included in the area of CamShift computed result and this area is among a certain distribution ${ }^{[6]}$. We can judge that the tracking is right. Then computation is made further using CamShift algorithm and the computed result as an observed value update Kalman filter. Otherwise, the computed result that hand tracking is abnormal using CamShift is effected by other colors. Additionally, we cannot predict the next frame's position accurately using CamShift to update Kalman filter ${ }^{[4]}$, which result in failing to track. We utilize the predicted value computed by Kalman filter as the initialized searching window of CamShift algorithm and figure out the accurate hand position. Then we utilize the position value to update Kalman filter. Aa a result, we can obtain the precise tracking result.

\section{Experimental Results and Analysis}

In order to verify the validity of the algorithm, in this paper, we use the Opencv library function to compile program in Windows 7 system. The video images used in the experiment is $640 * 480$.

\section{Hand Detection Experiment}

The figure 2 is the experiments and results of the initial search window, which is generated by skin color detection and inter-frame difference, (a) is the first frame, set it as the background image. (c) is the binary image generated by skin color detection of the first frame, which many color is close to the skin color is also identified as skin color. Then every frame after the first frame will use inter-frame difference algorithm to calculate with the first frame for the differential.In the experiment,we set the threshold to 1500 , when the number of white pixels is greater than 1500 by inter-frame difference,we will stop judging and process the difference image. In the experiment, the number of white pixels exceeds the threshold in the twenty-fifth frame. (b) is the image when it is the twenty-fifth frame, (d) is the binary image generated by skin color detection and inter-frame difference. (e) is the image that remove the skin color part in the background image, There are also some error points caused by illumination and some other factors in the binary image.(f) is the position of hand in the image after erode the image.

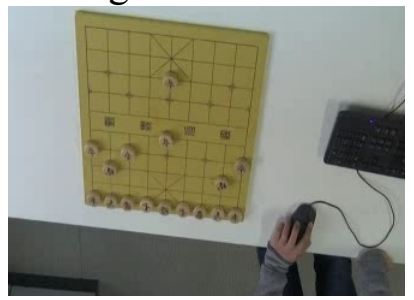

(a) The first frame image

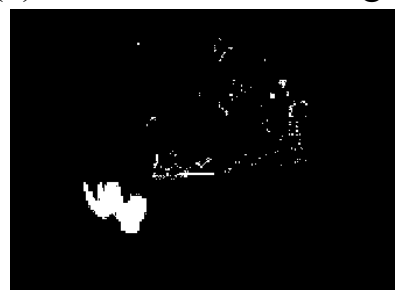

(d) Inter-frame difference

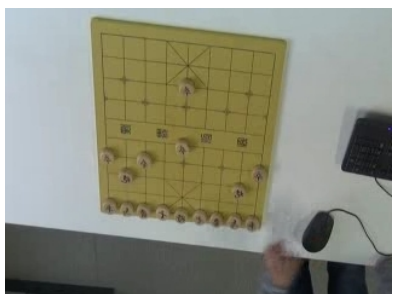

(b)

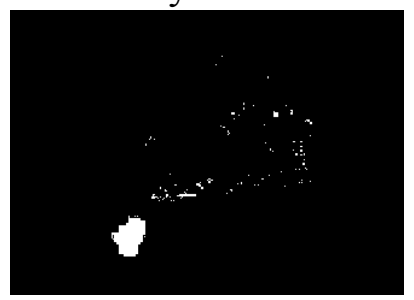

(e) Remove the background image

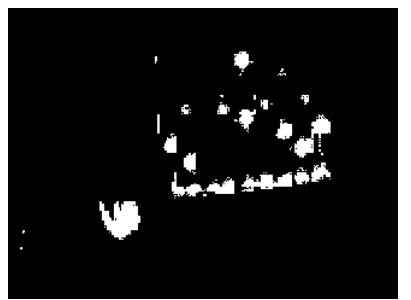

(c) Skin color detection

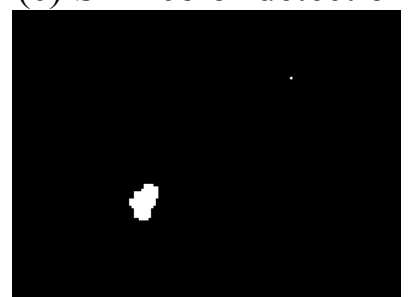

(f) The position of hand

Fig. 2 Initialize the search window

The skin color information in (f) can be used as the initial search window in CamShift algorithm. Then,we can track the position of hand automatically and quickly.

\section{Hand tracking experiment}

The figure 3 is the color histogram generated by the hand region after skin color detection and inter-frame difference. (a) is the color histogram generated by eight bins, (b) is the color histogram generated by 36 bins. In practice, we use 36 bins to ensure the accuracy of the tracking. The image can be converted to a color probability distribution by using the histogram. 


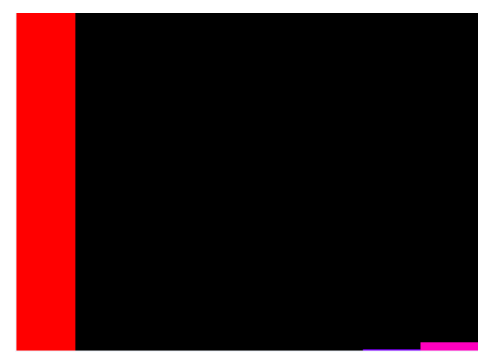

(a) 8 bins

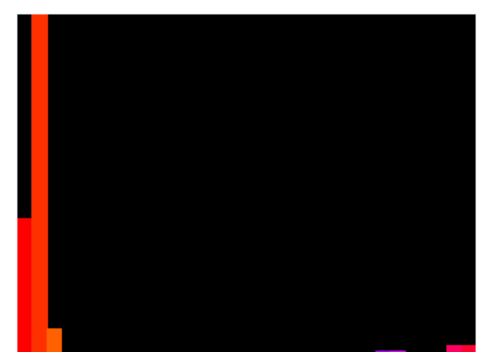

(b) 36 bins

Fig. 3 The color histogram generated by the hand region

The figure 4 is the effect drawing by use of CamShift algorithm and Kalman filter, The area in the ellipse is the area of the hand,and the point in the ellipse is the position of hand predicted by Kalman filter.When the area of the ellipse is within a range and the point is in the ellipse, use CamShift algorithm for tracking,(a) and (c) is the impression drawing.(b) and (d) is the color probability distribution when tracking the hand.

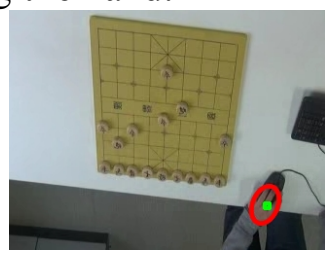

(a)

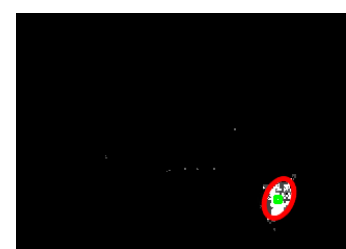

(b)

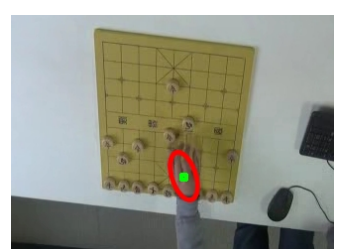

(c)

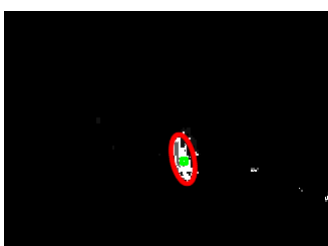

(d)

Fig. 4 Tracking experiments

CamShift algorithm can track the hand accurately. The figure 5 are the questions in the experiment.(a) is the image generated by the color histogram that use 8 bins to track. In the first image,there is no color interference, so the tracking is normal,but when the position of hand is in the checkerboard,the area of hand position increase greatly due to color interference.Increase the number of color space is a good solution to this problem.(b) is the image tracking lost.In this picture, because of the color of the piece, The result of this CamShift algorithm identify the position of the chess piece as the hand.The position predicted by Kalman filter is in the hand region. This time,we can detect whether the tracking is correct according to the prediction point is in the elliptical region.If the prediction point is not in the elliptical region,we can initialize the search window by use of the prediction region of Kalman filter,and then use CamShift for tracking.

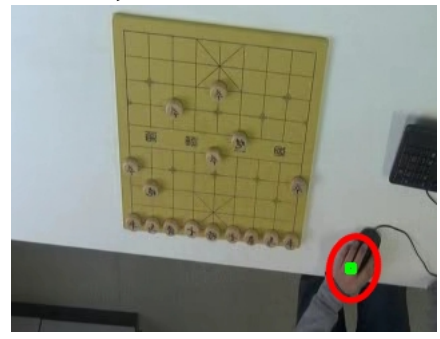

(a) The result by 8 bins

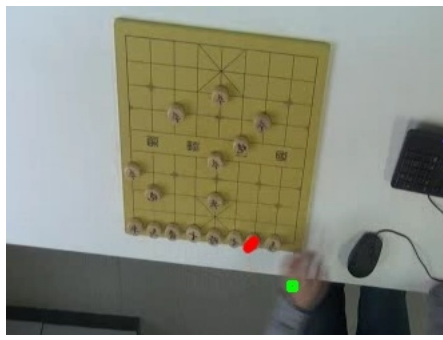

(b) Tracking lost

Fig. 5 Tracking questions in the experiment

\section{Conclusion}

In this paper, CamShift algorithm is used to track hand in the chess game system.Due to the limitations of the algorithm in practical application, an algorithm based on skin color detection and inter-frame difference is used to detect the position of hand and this position is the initial search window in Camshift.This algorithm can detect and track the position of hand automatically.Kalman filter is used to predict to correct tracking lost and improve the accuracy of tracking. The results show that the improved algorithm in this paper can detect and track the hand accurately and real-timely.

But,The premise of the algorithm is that the hand must keet in the scene.How to judge when the hand leave and return to the scene need to study further. 


\section{References}

[1] Michael J. Jones,James M. Rehg. Statistical Color Models with Application to Skin Detection[J]. International Journal of Computer Vision . 2002 (1)

[2] Ing-Sheen Hsieh,Kuo-Chin Fan,Chiunhsiun Lin.A statistic approach to the detection of human faces in color nature scene. Pattern Recognition . 2002

[3] Garcia C and Tziritas G.Face detection using quantized skin color regions merging and wavelet packet analysis. IEEE Trans.on Multimedia . 1999

[4] Panahi R,Gholampour I,Jamzad M.Real time occlusion handling using Kalman filter and mean-shift. The 8th Iranian Conference on Machine Vision and Image Processing . 2013

[5] Amir Hooshang Mazinan,Arash Amir-Latifi. Applying mean shift, motion information and Kalman filtering approaches to object tracking[J]. ISA Transactions . 2012 (3)

[6] Chu Hong-xia,YE Shu-jiang, Guo Qing-chang,et al.Object Tracking Algorithm Based on Camshift Algorithm Combinating with Difference in Frame. IEEE International Conference on Automation and Logistics . 2007

[7] Yang Bo,Zhou Hong-jun,Wang Xue.Target tracking using predicted Camshift. Proceedings of the World Congress on Intelligent Control and Automation . 2008 